\title{
CAMBIOS EN EL PERFIL BIOQUÍMICO HEPÁTICO DE ALPACAS POSITIVAS A HUEVOS DE Fasciola hepática
}

\section{CHANGES IN LIVER BIOCHEMICAL PROFILE IN POSITIVE ALPACAS TO} EGGS F. hepatica

*José Rodríguez G.1, Manuel Barrios-Arpi ${ }^{2}$, Bernardo Lopez-Torres ${ }^{3}$; Juan Lucas L.1, Carlos Arana1, Gonzáles-Gustavson E. ${ }^{1}$, Juan Espinoza B. ${ }^{3}$, Eva Casas A. ${ }^{4}$ y Heidi Ceferino J. ${ }^{5}$ ${ }^{1}$ Centro Experimental IVITA-Mantaro, ${ }^{2}$ Laboratorio de Patología Clínica, ${ }^{3}$ Laboratorio de Farmacología y Toxicología Veterinaria, ${ }^{4}$ Laboratorio de Parasitología Veterinaria. Facultad de Medicina Veterinaria, Universidad Nacional Mayor de San Marcos FMV-UNMSM. Av. Circunvalación 2800 San Borja-Lima-Perú, ${ }^{5}$ SENASA, Región Pasco-Perú.

*Correspondencia al autor: joserodriguezmv@gmail.com

\section{RESUMEN}

El objetivo del presente estudio fue determinar cambios en el perfil bioquímico hepático en alpacas con presencia de huevos de F. hepatica en heces. Se utilizaron muestras de suero y heces de 35 alpacas machos de entre 2 y 5 años de edad, de los cuales 18 fueron positivos y 17 negativos al examen coproparasitológico (sedimentación) para identificación de huevos de F. hepática. Se observó diferencia significativa $(\mathrm{p}<0.05)$ entre los valores de ALT, GGT y bilirrubina directa entre los animales positivos y negativos. Concluimos que la presencia de huevos de Fasciola hepatica guarda relación con la elevación de algunos parámetros del perfil hepática posiblemente por alguna alteración en el parénquima producto de una infección crónica en alpacas.

Palabras clave: Huevos de F. hepática, alpacas, perfil bioquímico hepático

\section{ABSTRACT}

The aim of this study was to determine changes in the liver biochemical profile in alpacas with presence of F. hepatica eggs. We used serum and feces samples of 35 male 
alpaca between 2-5 years old, 18 were positives and 17 were negatives in coproparasitologic analysis for F. hepatica eggs. Differences are showed $(\mathrm{p}<0.05)$ in the values of ALT, GGT and direct bilirubin between positives y negatives animals. We concluded that there is relationship between presence of F. hepatica eggs and high levels in the liver biochemical profile, maybe due to alterations in the hepatic parenchyma by chronic infection in alpacas.

Keywords: F. hepatica eggs, alpaca, liver biochemical profile

\section{INTRODUCCIÓN}

La infección por Fasciola hepatica es un serio problema en la producción alpaquera por la disminución de la productividad de los animales y por la alta mortalidad que ocasiona en animales que no son tratados (Leguía y Casas, 1999). La fasciolosis se presenta tanto en zonas de baja como de elevada altitud de Bolivia, Perú, Ecuador y Venezuela (Mas-Coma et al., 2001). Londoñe et al. (2009) hallaron en años recientes, formas larvarias de Fasciola hepatica en caracoles Lymnaeidae por encima de los $4000 \mathrm{msnm}$, probablemente debido al incremento de las temperaturas en zonas alto andinas, usualmente muy frías, causado por el calentamiento global (Mas-Coma et al., 1999).

En el Perú es una enfermedad endémica en la ganadería, y responsable de cuantiosas pérdidas en la industria pecuaria. Además, puede afectar al hombre y es considerada como una enfermedad zoonótica de importancia en la salud pública (Ministerio de Salud Pública, 1991/1995). Esta enfermedad es muy prevalente por toda la región altonadnina del Perú, donde se encuentra la mayor cantidad de alpacas del mundo (Neyra et al., 2002). La transmisión de la infección es facilitada por la gran carga parasitaria que contamina las pasturas con la forma infectiva, así mismo el clima y la falta de programas de control contra favorecen el ciclo del parásito.

Las larvas enquistadas del parásito trematodo, F. hepatica, puede infectar a mamíferos como vacas, ovejas, carnívoros y los seres humanos (Mas-Coma et al., 1999). Después eclosionan las formas juveniles en el intestino y migran hacia el hígado, donde maduran a la forma adulta del tremátodo (Andrews, 1999). La infección por fasciolasis pueden causar daños graves en el hígado y, en una etapa posterior, a la bilis con importantes consecuencias sistémicas como la anemia (Behm, 1994). En el ganado vacuno y ovino la fasciolasis causa importantes pérdidas económicas en la industria y su control actual se basa principalmente en 
el uso de fármacos antihelmínticos y manejo de pastos. Sin embargo, la $F$. hepatica se ha vuelto drogo-resistente (Gaasenbeek et al., 2001), y el uso de medicamentos anti-helmintos tiene inconvenientes para el medio ambiente y la calidad de los alimentos. La vacunación es vista como una alternativa no química para el control de la fasciolosis (Dalton y Mulcahy, 2001; Smith, 1999).

A pesar de la importancia de esta infección por el impacto negativo que ocasiona en la calidad y productividad de la fibra de alpaca, lo que se conoce la patología hepática y los factores que favorecen y están asociados a la patogénesis son poco conocidos. La susceptibilidad del huésped al tremátodo es un característica de esta enfermedad, siendo por ejemplo el ganado bovino capaz de aumentar su respuesta inmune para inmunoprotegerse en subsecuentes infecciones, por su parte la oveja desarrolla poca o ausente respuesta inmunoprotectiva a la infección por F. hepatica (Mulcahy et al., 1999). No se sabe si las alpacas llegan a hacer resistencia después de una primera infección a $F$. hepatica. Sin embargo, los exámenes sugieren que las alpacas se vuelven a reinfectar frecuentemente en áreas endémicas (Neyra et al., 2002).

El presente estudio tuvo por objetivo determinar los cambios en el perfil hepático en alpacas positivas a huevos de Fasciola hepatica.

\section{MATERIALES Y MÉTODOS}

\section{Animales}

El estudio incluyó suero y heces de 35 animales aparentemente sanos del Matadero Municipal de Huancavelica-Perú, lo cuales provienen de crianza extensiva de la zona altoandina de dicha localidad. El rango de las edades iba de 2 a 5 años. Los animales fueron divididos en dos grupos positivos $(\mathrm{n}=18)$ y negativos $(\mathrm{n}=17)$ a la presencia de huevos de $F$. hepatica en heces.

\section{Muestras de sangre y análisis bioquímico}

Antes del sacrificio de los animales, se tomaron muestra de sangre para la bioquímica del perfil hepático de la vega yugular en tubos de $5 \mathrm{~mL}$ sin anticoagulante. Los tubos fueron 
centrifugados a $3000 \mathrm{rpm}$ por 20 minutos para la separación del suero, e inmediatamente el suero fue almacenado en nitrógeno líquido a $-196^{\circ} \mathrm{C}$ y transportados para su análisis de laboratorio. Los parámetros evaluados fueron: bilirrubina total (BT), bilirrubina directa (BD), proteínas totales (PT), albúmina (A), Aspartato aminotransferasa (AST) alanino aminotransferasa (ALT), gamma-glutamyl transferasa(GGT), fosfatasa alcalina (FA), usando los kits comerciales para cada parámetro (Far Diagnostics, Italia), siguiendo el protocolo del fabricante. La lectura se hizo por medio de analizador semiautomático BS3000P (SINNOWA, China).

\section{Muestras de heces y análisis coproparasitológico}

Las 35 muestras de heces fueron tomadas post-mortem y colectadas en bolsas de polietileno de autocierre y colocadas en cajas de poliestireno expandido con geles refrigerantes, para su posterior análisis.

Para la evaluación coproparasitológica se utilizó la técnica de sedimentación espontánea (Tello y Canales, 2000), donde se usó 4g de heces y se hizo un homogenizado en $10 \mathrm{ml}$ de solución salina hasta que se logró una suspensión adecuada. La mezcla fue vertida en un tubo cónico de plástico de $13 \times 2.5 \mathrm{~cm}$, de $50 \mathrm{ml}$ de capacidad filtrándola a través de gasa. Se completó el volumen del tubo con solución salina y se tapó herméticamente. Se agitó enérgicamente por 30 segundos y se dejó reposar por 45 minutos. Se eliminó el sobrenadante y con una pipeta se tomó una muestra del fondo del tubo. Se colocaron 4 gotas en dos láminas distintas, agregándole luego gotas de lugol y de solución salina a cada una. Finalmente, las láminas portaobjetos fueron cubiertas con laminillas y se observó al microscopio (100X y 400X).

\section{Análisis estadístico}

Para observar si hubo diferencias significativas entre los valores obtenidos de los animales positivos y negativos a huevos de F. hepatica, estos fueron analizados por el TStudent test con un nivel de confianza del 95\%. Los resultados se expresan como Prom \pm DS (Promedio \pm Desviación estándar). 


\section{RESULTADOS Y DISCUSIÓN}
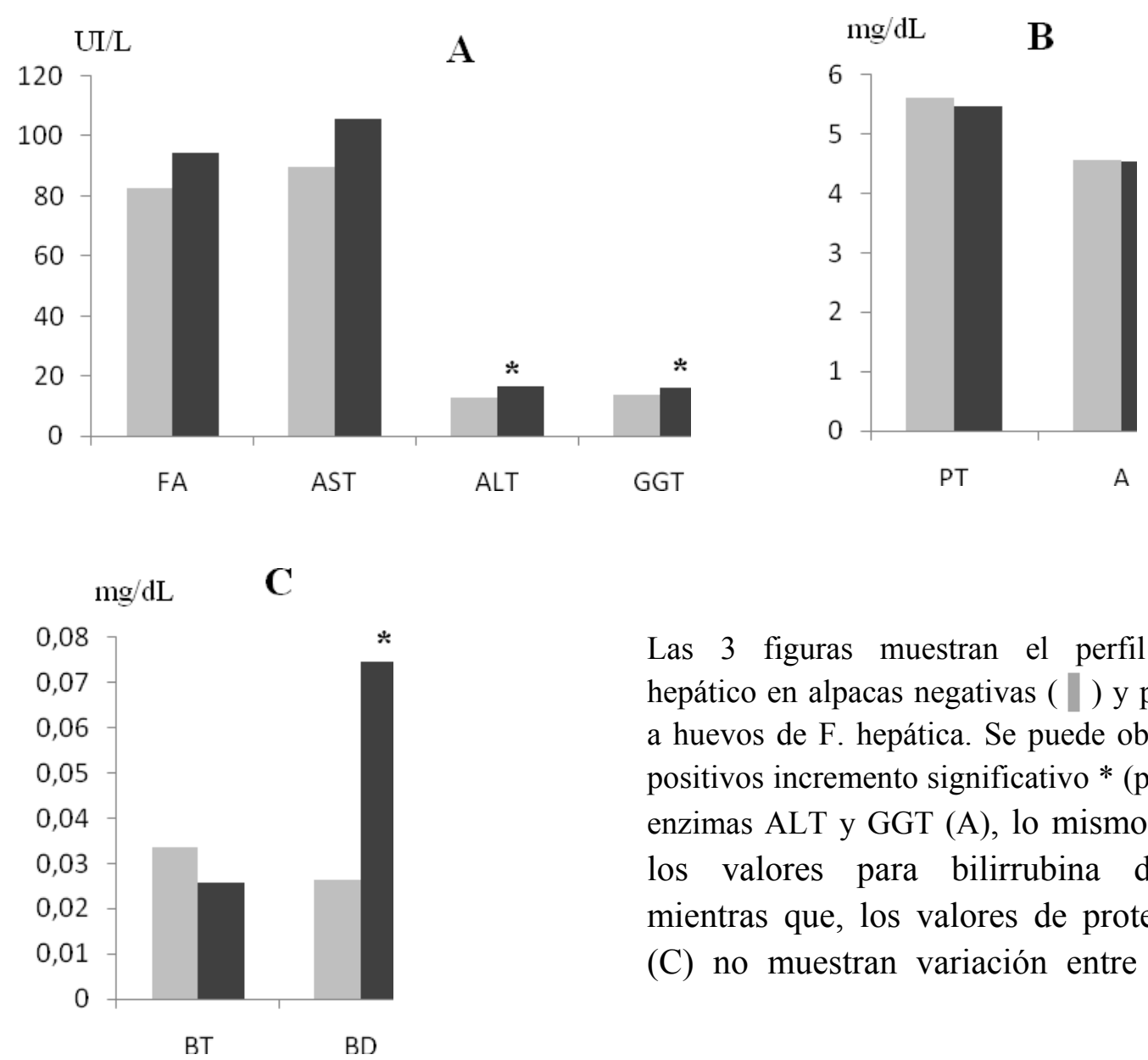

Las 3 figuras muestran el perfil bioquímico hepático en alpacas negativas ( \) y positivas ( \) a huevos de F. hepática. Se puede observar en los positivos incremento significativo $*(\mathrm{p}<0.05)$ en las enzimas ALT y GGT (A), lo mismo sucede con los valores para bilirrubina directa (B); mientras que, los valores de proteínas totales (C) no muestran variación entre positivos y

La fasciolasis o distomatosis es una enfermedad parasitaria de distribución mundial causal de pérdidas en los productos del ganado, en los costos asociados como tratamiento, la baja ganancia de peso, la reducida fertilidad y productividad, entre otros (Leguía, 1997; Rojas, 2004; Espinoza et al., 2010). Las duelas juveniles al recorrer hacia los conductos biliares para su posterior desarrollo a estadío adulto ocasionará daño a nivel del parénquima hepático así como en los canalículos biliares generando colestasis intrahepática para finalmente producir daño a nivel de los conductos biliares produciendo colestasis extrahepática debido al proceso inflamatorio y obstructivo reflejándose esta acción en un incremento plasmático de los niveles de enzimas que se localizan en los hepatocitos y el epitelio biliar (Anderson, 2002; Leguía y Casas, 1999). 
Los resultados obtenidos en el presente estudio muestran una diferencia estadísticamente significativa en los niveles de bilirrubina directa, alanina aminotransferasa (ALT) y Gamma glutamil transferasa (GGT), las cuales estarían asociadas con la acción que ejercen los parásitos adultos una vez alojados en los conductos biliares, considerando y relacionando la presencia de parásitos adultos en el animal con la presencia de huevos en las heces, ya que esto reflejaría una infección parasitaria crónica (Meyer y Harvey, 1998; Anderson, 2002).

La bilirrubina es un pigmento que se encuentra en la bilis que al estar incrementado en el plasma podría ocasionar la pigmentación de los tejidos, lo cual es un signo clínico denominado ictericia. Entre las causas de hiperbilirrubinemia tenemos las anemias hemolíticas y enfermedades hepatobiliares las cuales pueden conllevar al desarrollo de los tres tipos de ictericias que se conocen (pre hepática, hepática y post hepática) (Lattimer et al., 2005; Kaneko, 2008).

En general, las principales causas de incremento de enzimas hepáticas y bilirrubina en animales mayores son por anemias hemolíticas más que enfermedades hepáticas (Benjamín, 1991). En la enfermedad hepática crónica, las concentraciones de bilirrubina total se encuentran dentro de los límites normales para la especie e inclusive, se sabe que en casos de enfermedad hepática severa en bovinos adultos no se evidencia incremento de las concentraciones de bilirrubina, incrementándose solo ligeramente en novillos, cabras y ovejas (Lattimer et al., 2005). Estos datos, coinciden con los resultados del presente estudio donde no se evidenció elevación de la bilirrubina total (la cual se incrementa por elevación de la bilirrubina directa, indirecta o ambas), sin embargo si hubo diferencia significativa en los valores de bilirrubina directa para ambos grupos, lo cual podría tener dos posibles explicaciones: por una disminución del tiempo de supervivencia de los eritrocitos por una enfermedad crónica y por la acción mecánica de los parásitos adultos a nivel de los conductos y/o canalículos biliares. La enfermedad crónica o enfermedad inflamatoria puede ocasionar secuestro de hierro por parte de los macrófagos y los neutrófilos disminuyendo la síntesis de hemoglobina necesaria para la producción y maduración de glóbulos rojos como consecuencia la anemia de tipo no regenerativa y disminución de la vida media de los eritrocitos por deficiencia de hierro y la posterior hipoxia tisular en la cual los hepatocitos iniciarán el proceso de muerte celular con la tumefacción celular y por consecuencia el estrechamiento de los canalículos biliares con la regurgitación de la bilirrubina conjugada por 
hepatocitos funcionales (Sodikoff, 1996; Kaneko, 2008). La otra causa de elevación de la bilirrubina conjugada se explicaría por el efecto mecánico producido por los parásitos adultos, los cuales, al estar alojados en los conductos biliares ocasionarían un proceso inflamatorio debido a la lesión de dichos conductos y así también una obstrucción de los conductos biliares (colestasis extrahepática) debido a la acumulación de las duelas (Anderson 2002; Rojas, 2004). Sin embargo, ninguno de estos procesos elevó los niveles de bilirrubina conjugada por encima de sus valores normales, posiblemente debido a la poca severidad e intensidad de la respuesta inflamatoria asociada a la presencia de parásitos y a la obstrucción parcial que pudieran estar ejerciendo las duelas a nivel de los conductos biliares (Lattimer et al., 2005; Kaneko, 2008).

Otro de los parámetros que mostró diferencia estadística significativa entre ambos grupos fue la alanina amino transferasa (ALT). La ALT es una enzima de alta especificidad, concentrándose y mostrando una alta actividad en los hepatocitos, encontrándose a nivel del citosol. Cualquier alteración a nivel de membrana (o citosol) de los hepatocitos provocará la liberación de esta enzima al plasma sanguíneo incrementando sus niveles por encima de sus valores normales (Benjamín, 1991). Se sabe que en especies como canino, felino, primate y otras especies menores la actividad de ALT en hígado es muy alta, sin embargo, en bovinos, caprinos, ovinos, equinos y camélidos sudamericanos la actividad de ALT hepática es muy baja, es por ello que la ALT no es utilizada en el diagnóstico laboratorial de enfermedad hepática en animales mayores, siendo evaluada junto a alanina aminotransferasa (AST) y la creatin kinasa (CK) en el diagnóstico de patologías musculares (Kaneko, 2008). Es probable que en el presente estudio un mínimo incremento de ALT se pueda observar en casos de lesión de hepatocitos por la acción detergente que ejercen los ácidos biliares en el parénquima hepático producto de la obstrucción parcial de los conductos biliares por los parásitos adultos, ya que al haber mecanismos de obstrucción de los conductos biliares sean intra o extrahepáticos, esto provoca la regurgitación de acidos biliares los cuales actuarían como detergente y provocando una acción subletal a nivel de los hepatocitos traduciéndose en una alteración a nivel de la membrana, citosol y en raros casos a nivel de mitocondria conduciendo al incremento de los niveles de enzimas hepáticas, sin embargo al no haber una actividad enzimática elevada de ALT a nivel de los hepatocitos en animales mayores (incluyendo camélidos sudamericanos), no se evidenció una actividad tan elevada que supere los niveles normales de ALT (Siguas et al., 2007; Flores, 2009; ISIS, 2009). 
La enzima Gamma glutamil transferasa (GGT) es una enzima de moderada especificidad que se encuentra principalmente en células con alta secreción o absorción presentes en el hígado, riñón, páncreas y el intestino. La GGT es considerada un marcador sérico principalmente para enfermedades del sistema hepatobiliar asociado a colestasis y en el diagnóstico de enfermedades hepáticas en la mayoría de animales (Kaneko et al., 2008) presentando valores referenciales por encima de los valores de pequeños animales (Braun et al., 1983; Milne y Doxey, 1985). Por lo tanto, la GGT indica fibrosis hepática al migrar los parásitos a los conductos biliares, donde alcanzan el estado adulto en ovinos, camélidos y bovinos (Boray 1982). Estos datos coinciden con los resultados obtenidos en el presente estudio donde los niveles de GGT fueron mayores en el grupo positivo a huevos de Fasciola hepática, sin embargo, estos valores se encontraron dentro de los límites normales para la especie, lo cual podría explicarse en base a la baja carga parasitaria y como consecuencia un menor efecto obstructivo por parte de los parásitos adultos en los conductos biliares. Algunos autores consideran la GGT como la enzima de mayor sensibilidad de enfermedad hepática en animales mayores, incrementándose en los primeros días post daño hepático manteniéndose elevado hasta las fases finales, esto, debido a debido a su excreción urinaria y láctea y a la rara presentación de pancreatitis en animales mayores (Kaneko, 2008). Al haber baja carga parasitaria en los conductos biliares habrá poca inducción de esta enzima a este nivel reflejándose en un incremento ligero de la GGT manteniéndose en los límites normales para la especie (ISIS, 1999; Flores, 2009).

La Aspartato aminotransferasa (AST) es una enzima de moderada especificidad que se encuentra concentrada y en alta actividad en músculo esquelético y cardiaco, cerebro y ertirocitos, localizándose en su mayoría en las mitocondrias y en menor proporción a nivel del citosol. La AST no es específica de hígado, sin embargo, es utilizada ampliamente en animales mayores para el diagnóstico de enfermedades hepatobiliares, así como en trastornos musculares. En el presente estudio no se encontraron diferencias significativas de los niveles de AST entre ambos grupos, presentando niveles dentro de los valores de referencia encontrados en otros estudios en esta especie (Flores, 2009; ISIS, 2009), lo cual podría explicarse por la ausencia de daño letal en los hepatocitos (necrosis celular) y consecuente elevación de AST a partir de las mitocondrias. Otra enzima que se mantuvo dentro de sus valores de referencia fue la Fosfatasa alcalina (ALP), considerada de baja especificidad en la mayoría de especies ya que se presenta una actividad elevada en muchos tejidos del organismo, siendo ésta observación una limitante para su uso amplio en Medicina Veterinaria 
(Benjamín, 1991; Kaneko, 2008), y asimismo otra de las limitantes para su uso como marcador hepático en animales mayores es el amplio rango referencial que se maneja para este grupo de animales (Siguas et al., 2007).

Finalmente, con respecto a los valores de proteínas totales, no se encontró diferencia significativa entre ambos grupos, probablemente debido a la intensidad del daño de los hepáticos, lo que conllevaría a que los niveles de proteínas tales como proteína de fase aguda u otras proteínas sintetizadas por los hepatocitos no se incrementen en casos de faciolasis, manteniéndose dentro de sus valores referenciales para ambos grupos estudiados (Kaneko, 1989; ISIS, 2009; Flores, 2009).

\section{CONCLUSIONES}

- Los valores de ALT, GGT y bilirrubina directa se encuentran incrementados en alpacas positivas a huevos de Fasciola hepática, sin embargo, estos valores no superaron los valores referenciales para la especie.

- No se observaron diferencias significativas entre los niveles de AST, fosfatasa alcalina, bilirrubina total, bilirrubina indirecta y proteínas totales entre alpacas positivas y negativas a huevos de Fasciola hepática.

- Los cambios observados en los niveles de enzimas hepáticas y proteínas totales fueron similares a los encontrados en otros estudios realizados en rumiantes, sin embargo, se evidenció un incremento de los niveles de bilirrubina en los animales de estudio en relación a otros rumiantes.

Agradecimientos

Este trabajo fue ejecutado por la Facultad de Medicina Veterinaria de la Universidad Nacional Mayor de San Marcos y con fondos provenientes del Proyecto $\mathrm{N}^{\mathrm{o}} 173-$ FINCyT-IB-2013, Lima-Perú.

\section{BIBLIOGRAFÍA}

Anderson D. 2002. Liver disease in camelids. The Ohio State University - USA. [Internet],[15marzo2008].Disponible in: http://www.rmla.com/LiverDisease.htm. 
Andrews SJ. 1999. The life cycle of Fasciola hepatica. En: Dalton JP, ed. Fasciolosis. Wallingford (Reino Unido): CABI Pub. p 1-29.

Behm CA. 1994. Pathophysiology of Fasciola hepatica infections in mammals. En: Boray ed. Immunology, pathology and control of fasciolosis. Izmir: MSD AGVET.

Benjamin M. 1991. Manual de patología clínica en veterinaria. México: Ed. Limusa. 421 p.

Boray JC. 1982. Chemotherapy of fasciolosis. New South Wales Vet. Proc. 18, 42-47

Braun JP; Bernard P; Burgat V; Ricop AG. 1983. Gamma glutamyl transferase in domestic animals. Vet Res Commun 6, 77-90.

Dalton JP, Mulcahy G. 2001. Parasite vaccines-a reality?. Vet Parasitol., 98(1-3):149-67.

Espinoza JR, Terashima A, Herrera-Velit P, Marcos LA. 2010. Fasciolosis humana y animal en el Perú: impacto en la economía de las zonas endémicas. Rev Peru Med Exp Salud Pública 27(4): 604-612.

Gaasenbeek CP, Moll L, Cornelissen JB, Vellema P, Borgsteede FH. 2001. An experimental study on triclabendazole resistance of Fasciola hepatica in sheep. Vet Parasitol., 95(1):37-43.

Flores S. 2009. Determinación del perfil bioquímico sanguíneo hepático y renal en alpacas (Lama pacos) aparentemente normales. Tesis de Médico Veterinario. Lima: Univ. Nacional Mayor de San Marcos. 86 p.

Fowler ME. 2010. Medicine and surgery of camelids. $3^{\circ}$ ed. Willey Blackwell. 630 p.

Kaneko J. 1989. Serum proteins and dysproteinemias. In: Kaneko JJ (ed). Clinical biochemistry of domestic animals. $4^{\circ}$ ed. California: Academic Press. p 142-164.

Kaneko J. 2008. Clinical Biochemistry of Domestic Animals. Academic Press. 832 p.

International Species Information System (ISIS). 1999. Reference ranges for physiological data values of alpaca (Lama pacos), llama (Lama glama) and guanaco (Lama guanicoe). USA. [Internet], [5 julio 2007]. Disponible en: http://www.worldzoo.org

Latimer KS, Mahafeey EA, Prasse KW. 2005. Patología Clínica Veterinaria. $4^{\text {o }}$ ed. Ed. Multimédica. España. 551 p.

Leguía PG.1997. Acute and subacute Fasciolosis of alpacas (Lama pacos) and treatment with triclabendazole. Trop Anim Health Prod 29(1):31-2. 
Leguía G, Casas E. 1999. Enfermedades parasitarias en camélidos sudamericanos. Lima: Editorial de Mar. 190 p.

Londoñe P, Chávez A, Lí O, Suárez F, Pezo D. 2009. Presencia de caracoles Lymnaeidae con formas larvarias de Fasciola hepatica en altitudes sobre los $4000 \mathrm{msnm}$ en la sierra sur del Perú. Rev Inv Vet Perú, 20: 58-65.

Mas-Coma MS, Esteban JG, Bargues MD. 1999. Epidemiology of human fascioliasis: a review and proposed new classification. Bull World Health Organ, 77 (4): 340-346.

Mas-Coma S, Funotsu IR, Bargues MD. 2001. Fasciola hepatica and Lymnaeid snails occurring at very high altitude in South America. Parasitology, 123: 115-127.

Meyer DJ, Harvey JW. 1998. Veterinary laboratory medicine. 2nd ed. USA: Ed. WB Saunders. 373 p. 18. [MINAG] Ministerio de Agricultura del Perú. 2007. Camélidos sudamericanos. Perú. [Internet], [15 mayo 2007]. Disponible en: http://www.minag.gob.pe/pecuaria/pec_real_camelidos.html

Milne EM, Doxey DL. 1985. Gamma glutamyl transpeptidase and its multiple forms in the tissues and sera of normal dogs. Res Vet Sci 39: 385-387.

Ministerio de Salud Pública. 1991/ 1995. Cuadro Epidemiológico Nacional. Ciudad de La Habana, Cuba. 195 p.

Mulcahy G, Joyce P, Dalton JP. 1999. Immunology of Fasciola hepatica infection. En: Dalton JP, ed. Fasciolosis. Wallingford (Reino Unido): CABI Pub. p 341-376.

Neyra V, Chavarry E, Espinoza JR. 2002. Cysteine proteinases Fas1 and Fas2 are diagnostic markers of Fasciola hepatica infection in alpacas (Lama pacos). Vet. Parasitol., 105: 2132.

Rojas CM. Nosoparasitosis de los rumiantes domésticos peruanos. 2da ed. Lima: Martegraf. 2004:41.

Siguas O, Páucar R, Olazábal J, San Martín F. 2007. Valores bioquímicos sanguíneos en alpacas en dos épocas del año en condiciones de Huancavelica: aportes al perfil metabólico de la especie. [Internet], [20 marzo 2008]. Disponible en: http://www.produccionbovina.com.ar/produccion_de_camelidos/147

Siguas_BIOQUIMICA.pdf

Smith WD. 1999. Prospects for vaccines of helminth parasites of grazing ruminants. Int J Parasitol, 29: 17-24. 
Sodikoff C. 1996. Pruebas diagnósticas y de laboratorio en las enfermedades de pequeños animales. $2^{\mathrm{a}}$ ed. España: Ed Mosby-Doyma. 405 p.

Tello R, Canales M. Técnicas de diagnostico de enfermedades causadas por enteroparasitos. Diagnostico 2000; 39(4):197-8. 